\title{
VHE observation of Mrk 421 with TACTIC during 2012; multiwavelength study
}

\author{
B Ghosal *, K K Yadav, K K Singh, R C Rannot, A K Tickoo, \\ P Chandra, H Bhatt, K K Gaur, A Goyal, H C Goyal, N Kumar, \\ P Marandi, M Kothari, S Sahayanathan, K Chanchalani, \\ N K Agarwal, V K Dhar, S R Kaul, M K Koul, R Koul, \\ K Venugopal, C K Bhat, C Borwankar, N Chouhan \\ Astrophysical Sciences Division, Bhabha Atomic Research Centre. \\ Mumbai - 400 085, India.
}

\begin{abstract}
The nearby $(z=0.031) \mathrm{TeV} \gamma$-ray emitter BL Lac object Mrk 421 has been observed with the TACTIC telescope for nearly 118 hours during 01 February to 26 April, 2012 (MJD 55958 - 56044). Detailed analysis reveals the detection of $529 \pm 76 \gamma$-rays with $6.9 \sigma$ significance above $850 \mathrm{GeV}$ energy threshold during the period of 15 - 26 March, 2012 (MJD 56001 - 56013). Nearly simultaneous multiwavelength observations of the source also indicate a comparatively higher flux state during this period (MJD 56001 - 56013). In this paper, we present the study of Mrk 421 during this period in very high energy (VHE) domain along with the results from high energy (HE), X-ray, optical and radio observations. We reproduce the spectral energy distribution (SED) of the source during this period using the synchrotron and synchrotron self Compton (SSC) process. The model parameters of the emission region of the source are found to be consistent with the values reported in the literature.
\end{abstract}

Key words: (Galaxies:) BL Lac objects: individual: Mrk 421, Methods: Cherenkov imaging technique, Gamma-rays: observation

\section{Introduction}

Blazars are the most extreme subclass of radio loud active galactic nuclei (AGN) and represent a dominant class of extragalactic $\gamma$-ray sources. These objects are

\footnotetext{
* Corresponding author.

Email address: bitan@barc.gov. in (B Ghosal).
} 
classified as BL Lac and flat-spectrum radio quasars (FSRQs) depending on the absence and presence of optical features. Their nonthermal radiation is strongly Doppler boosted, originating from relativistic jet of magnetized plasma which is aligned very close to the observer's line of sight [1]. The observed broadband SED of blazars is characterized by a double hump profile with first peak usually in the IR to X-ray range, and second one in between $\mathrm{MeV}$ to $\mathrm{TeV}$ energies [2,3]. The first peak is commonly interpreted as due to synchrotron radiation of relativistic electrons accelerated in the magnetic field of jets, while the origin of the second peak is still uncertain. In the leptonic scenario, the high energy peak is interpreted as inverse Compton (IC) scattering of either the synchrotron (SSC model) or external photons (External Compton (EC) model) or both by the same population of relativistic electrons [4-6]. Alternatively, the hadronic models explain the emission of very high energy (VHE) $\gamma$-rays via pion decay with subsequent synchrotron and/or IC emission from the decay products, or by synchrotron radiation from ultrarelativistic protons [7-11]. The broadband SED of BL Lacs are often reproduced by simple synchrotron and SSC model. On the other hand, the high $\gamma$-ray luminosity of FSRQs demand the inclusion of EC process also for successful reproduction of the SED [18].

The BL Lac Markarian 421 (Mrk 421; $z=0.031$ ) is the first extragalactic source discovered in the TeV energy range by Whipple collaboration in 1992 [12]. Since its detection, it is found to be one of the most active and brightest extragalactic source, hence mostly studied object in the VHE $\gamma$-ray domain. Mrk 421 has been studied by various ground based telescopes [17,20-29] and found to be highly variable source of VHE $\gamma$-rays. Several aspects of the VHE photon emission from the source including the correlations between intensity of X-rays, optical and $\mathrm{TeV} \gamma$-rays have been studied by $[13,14]$. These observations have revealed a variable nature of the sources in terms of both flux as well as spectrum, with a time scale as short as few minutes during flaring activities [15-17]. The variability timescales can be the most direct way to probe the dynamics operating in the jet plasma, in particular compact regions of shock acceleration [19]. For example, a bright TeV flare was detected at a flux level of $\sim 10$ times of Crab Nebula flux in May, 2008, reported by [16]. These $\mathrm{TeV}$ observations, complemented by a campaign of multiwavelength observations, showed flux variability in all wave bands except in radio band. Long term multiwavelength observations of Mrk 421 during November 2007 to February 2010 by ARGO, Swift, RXTE etc. showed that the source had undergone several flares during which a long term correlation between $\gamma$-ray and X-ray fluxes was also indicated [29,30]. Several multiwavelength campaigns were reported by MAGIC group with the multi-instrument collaboration, focused on the characterisation of different states of Mrk 421 [31,61]. Similar long term spectral and temporal study of different states were reported very recently by [72,71]. In February, 2010, a broad band outburst from Mrk 421 has been detected simultaneously by several telescopes including TACTIC [21,32-37]. More recently, the source has shown high $\gamma$-ray activities in July, 2012 and April, 2013. The $\gamma$-ray flare in 2012 was reported as an orphan flare for not showing any X-ray counterpart [38]. However, in 2013 the 
high activity of source was observed in X-ray, optical and radio waveband with $\gamma$ rays. The detailed study of the X-ray flare were reported by $[39,40]$. The correlation between radio and GeV flare during 2012 and 2013 were studied by [41].

In the present work, we report VHE $\gamma$-ray detection with TACTIC from Mrk 421 during 01 February - 26 April, 2012 (MJD 55958 - 56044). The source has been nearly simultaneously observed at radio with OVRO, optical by Steward Observatory, X-ray by Swift/XRT and HE by Fermi/LAT. We separately study the HE and VHE spectrum at comparatively higher flux state of the source during 15 - 26 March, 2012 (MJD 56001 - 56013) indicated in most of the energy bands. We characterize of the broadband SED with a simple one-zone SSC model involving synchrotron and SSC processes. The paper is organized as follows: Section 2 describes briefly TACTIC telescope. In section 3, we present the details of multiwavelength observations and data analysis procedure followed in our study. In section 4, the results in the different wavelength bands are presented. The SED obtained during the comparatively high-state and its theoretical interpretation is described in section 5. The discussion and conclusion of the present study are given in section 6 . Throughout the work, we use a cosmology with $\Omega_{m}=0.3, \Omega_{\Lambda}=0.7$ and Hubble constant $H_{0}=70 \mathrm{~km} \mathrm{~s}^{-1} \mathrm{Mpc}^{-1}$.

\section{TACTIC Telescope}

The TACTIC $\gamma$-ray telescope located at Mt Abu $\left(24.6^{\circ} \mathrm{N}, 72.7^{\circ} \mathrm{E}, 1300 \mathrm{~m}\right.$ asl $)$ Rajasthan, India, deploys a F/1 type tracking light collector of $\sim 9.5 \mathrm{~m}^{2}$ area. The light collector is made up of $34 \times 0.6 \mathrm{~m}$ diameter front-coated spherical glass facets which have been pre-aligned to produce an on-axis spot of $0.3^{\circ}$ diameter at the focal plane. A 349 pixel Photo-Multiplier Tube (PMT:ETL 9083UVB) based imaging camera with an uniform pixel resolution of $\sim 0.3^{\circ}$ which covers a total field of view of $\sim 6^{\circ} \times 6^{\circ}$ has been used to record images of atmospheric Cherenkov events. The data acquisition and control systems of the telescope [42] have been designed around a network of PCs running the QNX (version 4.25) real-time operating system. The single pixel threshold was set to 14 photo-electrons (pe) for nearest neighbor non-collinear triplets (3NCT) trigger logic [43]. The triggered events are digitized by CAMAC based 12-bit charge to digital converters (CDC) which have a full scale range of $600 \mathrm{pC}$. The sensitivity of TACTIC during the period of these observations was such that it detected VHE $\gamma$-rays from the Crab Nebula direction at $5 \sigma$ statistical significance level in 12 hours above $\sim 850 \mathrm{GeV}$. The data collected on Crab Nebula covers the zenith angle range $5^{\circ}$ to $45^{\circ}$. The instrumentation and other details of the telescope can be found in [44].

Major upgradation work was carried out for TACTIC during November - December, 2011, in terms of installing new compound parabolic concentrators (CPC) on the imaging camera of the telescope in order to increase its photon collection ef- 
ficiency. These CPCs have square entry and circular exit aperture and $\sim 85 \%$ reflectivity in the wavelength range $400-550 \mathrm{~nm}$. In addition, the trigger criteria were modified with the inclusion of nearest neighbour collinear triplets (NNCT), a dedicated CCD camera was installed for detailed pointing runs which gives source position with an accuracy of $\sim 3$ arc-min in the camera plane and old signal, high voltage cables were replaced with new ones in the upgraded system. More detail about the up-gradation of TACTIC can be found in [45].

\section{Multiwavelength Observations and Data Analysis}

Data obtained on Mrk 421 with TACTIC telescope during 01 February to 26 April, 2012 were complemented with the nearly simultaneous archival data from Fermi/LAT, Swift/XRT, Steward Observatory and OVRO for the multiwavelength study. The multiwavelength observations and corresponding data analysis procedures are described below.

\subsection{TACTIC data}

The VHE observations were performed with TACTIC telescope in tracking mode during 01 February to 26 April, 2012 (MJD 55958 - 56044) for $\sim 118.0$ hours covering the zenith angle range $12^{\circ}$ to $45^{\circ}$. The mean zenith angle for Mrk 421 during March, 2012 is $24^{\circ} \pm 8^{\circ}$, whereas for Crab Nebula it is $23^{\circ} \pm 10^{\circ}$, ensuring the same energy threshold for both the sources. Data quality checks were applied in order to select good quality data wherein we have used standard data quality checks like using inputs from atmospheric condition logs, compatibility of the prompt coincidence rates with the expected zenith angle behavior, Poisson distribution of prompt events and steady behavior of chance coincidence rates with time. After rejecting bad quality data runs, nearly 77.2 hours of data were retained for detailed analysis. Details of the observations considered for the detailed analysis are given in the Table 1.

While performing detailed analysis, firstly data were subjected to pedestal subtraction followed by the standard two-level 'image cleaning' [44] procedure with picture and boundary thresholds of $6.75 \sigma$ and $3.25 \sigma$, respectively. The image cleaning threshold levels were first optimized using the Crab Nebula data and then applied to the data presented in this paper. Further, all pixels were accounted for inter-pixel gain variation with respect to the one provided with a fixed high voltage. The clean Cherenkov images were characterized by calculating their standard image parameters such as length (L), width (W), distance (D), alpha $(\alpha)$, size $(\mathrm{S})$, frac2 (F2), asymetry (ASYM) etc. [47]. The L and $\mathrm{W}$ of the images increase with size and 
Table 1

Log of observations of Mrk 421 with TACTIC telescope during 2012 considered for analysis.

\begin{tabular}{|l|l|c|c|}
\hline \multirow{2}{*}{ Spell } & Observation Dates & \multicolumn{2}{|c|}{ Observation Time (hours) } \\
\cline { 3 - 4 } & & Spell wise & Total \\
\hline I & February 01, 02, 18 - 29, March 01. & 18.9 & \\
II & March 15 - 26. & 37.7 & 77.2 \\
III & April 15 - 24. & 20.6 & \\
\hline
\end{tabular}

therefore the dynamic super-cuts given in Table 2 have been applied to segregate the $\gamma$-rays from the background cosmic rays $[48,49]$. The $\gamma$-ray selection criteria based on imaging cuts have been obtained on the basis of Monte Carlo simulations carried out for the TACTIC. The cuts have been validated using data collected on the standard candle $\gamma$-ray source Crab Nebula using the TACTIC telescope. The next step in the data analysis chain deals with estimating the number of $\gamma$-ray events from the source direction in the presence of overwhelming background of cosmic ray events. For a single imaging telescope, this is done by plotting the frequency distribution of the $\alpha$ parameter (defined as the angle between the major axis of the image and the line joining the image centroid with the source position) after applying the dynamical super cuts except $\alpha$. Gamma rays coming from a point source have smaller values of the $\alpha$ parameter, while cosmic ray events, because of their isotropic nature, are uniformly distributed in all $\alpha$ values ranging from $0^{\circ}$ to $90^{\circ}$. For the TACTIC telescope, the $\alpha$ range for $\gamma$-ray events is defined from $0^{\circ}$ to $\leq 18^{\circ}$. The flat distribution of $\alpha$ in the range $27^{\circ} \leq \alpha \leq 72^{\circ}$ has been used to determine the background events present in the signal region. It is important to note that in one of our previously published work the background is estimated using the $\alpha$ range $27^{\circ}$ $\leq \alpha \leq 81^{\circ}$, leaving the last bin of the on-source $\alpha$ plot i.e. $81^{\circ} \leq \alpha \leq 90^{\circ}$, due to the truncation effect of the shower images as described in [27]. This effect has been observed to be extended upto last two bin for this data set under study. So we did not consider last two bins of the on-source $\alpha$-plot i.e. $72^{\circ} \leq \alpha \leq 90^{\circ}$ to estimate background in this case.

The number of $\gamma$-ray events are then calculated by subtracting the expected number of background events from the $\gamma$-ray domain events. Finally, the significance of the excess events has been finally calculated using the maximum likelihood method proposed in [51]. 
Table 2

Dynamic super cuts selection criterion used for analyzing the TACTIC data.

\begin{tabular}{lc}
\hline Parameters & Cuts Value \\
\hline L & $0.16^{\circ} \leq L \leq((0.1446+0.07179 \times \theta)+(0.0683-0.0375 \times \theta \times \ln S))^{\circ}$ \\
W & $0.04^{\circ} \leq W \leq((-0.1436+0.3217 \times \theta)+(0.0738-0.0790 \times \theta \times \ln S))^{\circ}$ \\
D & $\left(0.53 \times \cos ^{0.47} \theta\right)^{\circ} \leq \mathrm{D} \leq\left(1.14 \times \cos ^{-0.41} \theta\right)^{\circ}$ \\
F2 & F2 $\geq(0.29+0.1146 \times \theta)(\theta=$ zenith angle in degree $)$ \\
S & S $\geq 50$ pe \\
$\alpha$ & $\alpha \leq 18^{0}$ \\
L/W & L/W $\geq 1.55$ \\
AS & ASYM $\geq 0.0$ \\
\hline
\end{tabular}

\section{$3.2 \mathrm{Fermi} / \mathrm{LAT}$ data}

The Fermi/LAT data of Mrk 421 have been taken from the publicly available NASA database ${ }^{1}$. The LAT, on-board Fermi satellite, is a pair conversion detector [52] with a very wide field of view of 2.4 sr covering $\sim 20 \%$ of the sky at any instant with angular resolution of $\sim 1^{0}$ at $10 \mathrm{GeV}$. It is sensitive to $\gamma$-rays in the energy range $\sim 20 \mathrm{MeV}$ to $300 \mathrm{GeV}$ with peak effective area of $\sim 8000$ $\mathrm{cm}^{2}$ at $1 \mathrm{GeV}$. The data have been analyzed using the standard Fermi/LAT ScienceTools software package version $v 10 r 0 p 5^{2}$ and with the help of the analysis threads and other documentation available from the Fermi Science Support Center webpages ${ }^{3}$. We have selected only 'SOURCE' class events ( evclass $=128$ and evtype $=3$ ) in the energy range $100 \mathrm{MeV}$ to $300 \mathrm{GeV}$ within $15^{\circ}$ region of interest (ROI) centered around the source positions using gtselect task. In order to avoid background contamination from the bright Earth limb, we have discarded photons arriving from zenith angle greater than $90^{\circ}$. We selected only those time intervals during which the events/photons qualify for science analysis using the filter expression (DATA_QUAL $>0$ \&\& LAT_CONFIG==1) using gtmktime task. The livetime for the source was calculated by gtltcube task and exposure map of the source was generated using gtexpmap. We used the unbinned maximum likelihood fitting technique [53] with power-law model to constrain the spectral characteristics of Mrk 421 using gtlike task. The background model for this analysis was constructed by including nearby $\left(\leq 15^{\circ}\right.$ away) $\gamma$-ray sources from the third Fermi catalog (3FGL; [59]) keeping their spectral parameters fixed to the catalog values, except Mrk 421. Besides, gall_iem_v06.fits and iso_P8R2_SOURCE_V6_v06.txt were

\footnotetext{
$\overline{1 \text { http://fermi.gsfc.nasa.gov/ssc/data/access }}$

2 http://fermi.gsfc.nasa.gov/ssc/data/analysis/software/

3 http://fermi.gsfc.nasa.gov/ssc/data/analysis/
} 
used to consider a galactic diffuse component and an isotropic component, respectively in the background model. The spectral analysis was performed with the postlaunch instrument response function P8R2_SOURCE_V6. Finally, the power-law index and photon flux were estimated in energy range $0.1-300 \mathrm{GeV}$.

\subsection{X-ray data}

The Swift/XRT [68] is a space based grazing incidence Wolter I telescope with CCD detector covering the energy range of $0.2-10.0 \mathrm{keV}$ with effective area of $110 \mathrm{~cm}^{2}$ and field of view of 23.6 arc-min. The archival data of the source have been taken from the Fermi multiwavelength support program website ${ }^{4}$ in the time interval from 01 February to 26 April 2012 (MJD 55958 - 56044). The Swift/XRT data for the period 15 - 26 March, 2012 have been analyzed separately to derive the spectral features of the source using HEASoft package version 6.13. The observations were performed in windowed timing (WT) mode and the cleaned XRT event files with standard filtering criteria were produced using XRTPIPELINE version 0.12.6 with recent calibration files (version 20140709). The spectra of the source and background was generated from Xselect V2.4 $\mathrm{b}$ in energy band 0.3-10.0 $\mathrm{keV}$. The source photons were extracted from circular region with radius of 45 arcsec around source position. The nearby background with similar radius from the source free region was selected for individual observation according to the source position in the detector.

The ancillary response files (ARFs) were generated with the XRTMKARF task, applying corrections for the PSF losses and CCD defects using the cumulative exposure map. Finally the spectra were binned to have atleast 60 counts per spectral bin using GRPPHA. The spectra are fitted with power-law model with absorption due to a neutral hydrogen using XSPEC (ver 12.8.0) model PHABS $\times$ ZPOW for $z=0.031$. The line-of-sight absorption was fixed to a neutral hydrogen column density $\left(N_{H}\right)$ of $1.91 \times 10^{20} \mathrm{~cm}^{-2}$ (LAB survey; [67]). The values of derived parameters are given in Table 4. The energy fluxes were calculated in four sub-energy bands: 0.3 - $0.7 \mathrm{keV}, 0.7$ - $1.7 \mathrm{keV}, 1.7$ - $4.0 \mathrm{keV}, 4.0$ - 10.0 keV to derive the SED and to study the spectral evolution in that particular duration.

\subsection{Optical and Radio data}

The archival optical and radio data available in the Fermi multiwavelength support program websites ${ }^{56}$ were used in this multiwavelength study. The optical

\footnotetext{
${ }^{4} \mathrm{http} / / /$ www.swift.psu.edu/monitoring/

5 http://www.james.as.arizona.edu/ psmith/Fermi/

6 http://www.astro.caltech.edu/ovroblazars/index.php?page=home
} 


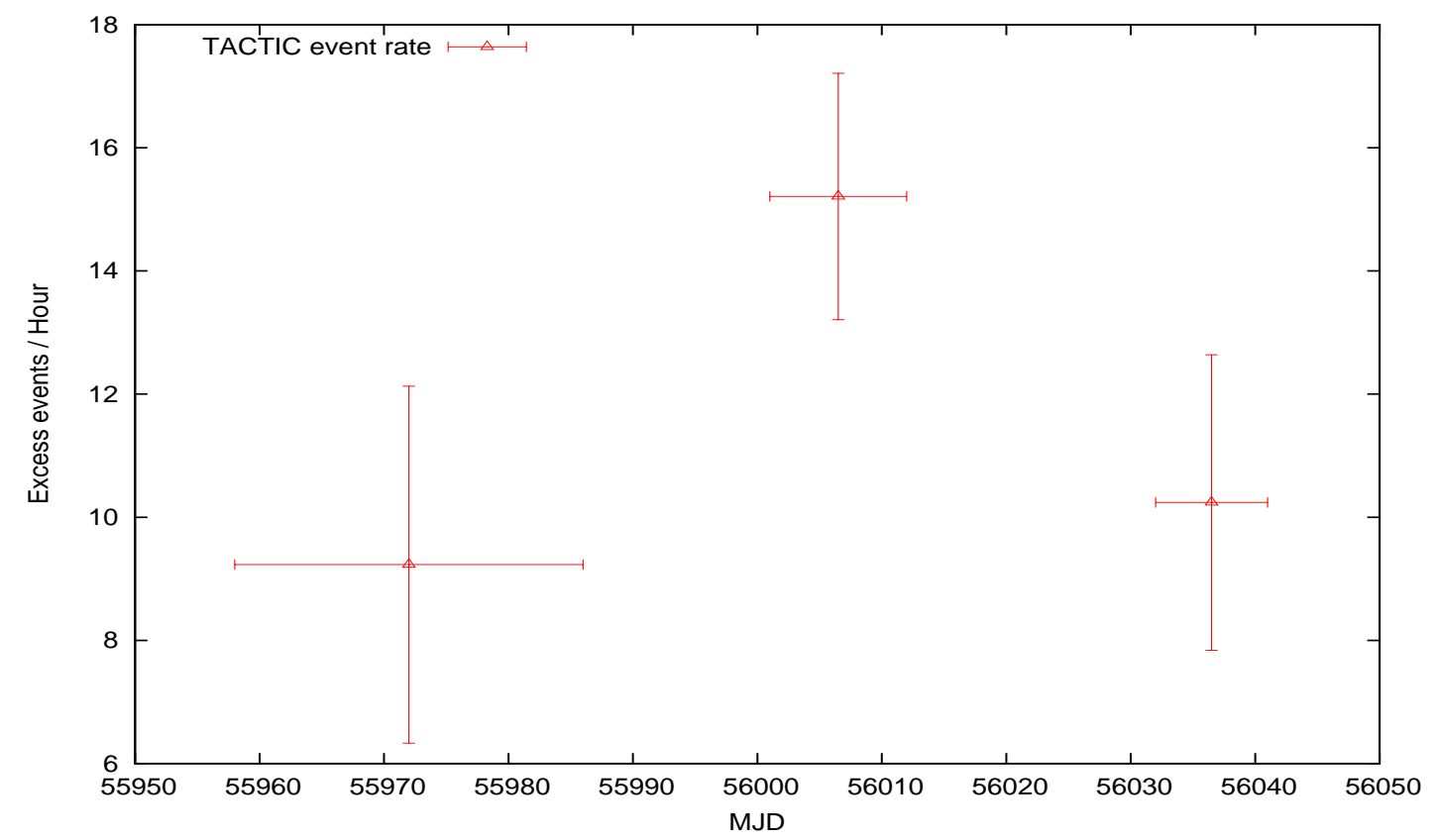

Fig. 1. The $\gamma$-ray rate per hour (excess event rate) detected by TACTIC during the three spell of observations as mentioned in the Table 1. It shows an enhancement of event rate of $\sim 5$ per hour during spell II (MJD 56001 - 56013) in comparison to other two spells.

observation were made using SPOL CCD Imaging/Spectropolarimeter at Steward Observatory [56]. We used optical V-band photometric data for this study. The $40 \mathrm{~m}$ single-dish radio telescope at Owens Valley Radio Observatory (OVRO) observed the source at $15 \mathrm{GHz}$ frequency. The telescope is equipped with dual-beamed off axis optics and a cool receiver installed at the prime focus. The systematic uncertainty is about $5 \%$ in the flux density. Analysis and calibration details are given in [60].

\section{Results}

\subsection{VHE results}

We have followed data analysis procedure discussed in the section 3.1, for analyzing Mrk 421 data recorded during 01 February to 26 April, 2012 (MJD 55958 - 56044) with the TACTIC $\gamma$-ray telescope for 77.2 hours. As evident from the Figure 1, the spell wise analysis shows an enhanced excess event rate for spell II. Therefore we analyzed this spell separately. During spell II which comprises of the period 15 - 26 March, 2012 (MJD 56001 - 56013), shows an excess of $529 \pm 76$ $\gamma$-ray like events with $6.9 \sigma$ significance in 37.7 hours.

Figure 2(a) shows the on-source $\alpha$-parameter distribution during 15 - 26 March, 


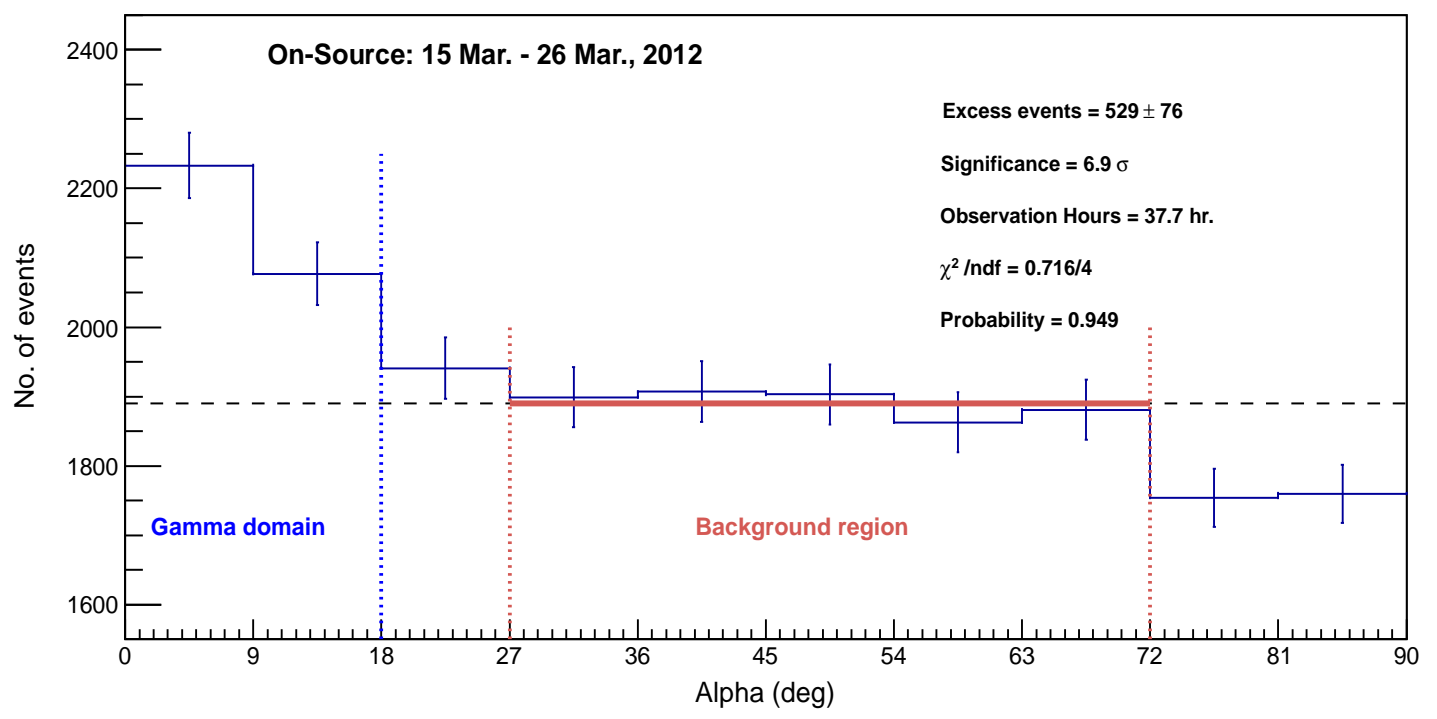

Fig. 2. The on-source $\alpha$-parameter distribution after applying the $\gamma$ domain cuts on the clean TACTIC data of Mrk 421 during 15 March to 26 March, 2012 (spell 2).

2012., whereas the nearly simultaneaous off-source distribution of $\alpha$-parameter during 19 March to 18 April, 2012, is shown in Figure 2(b). In both of the $\alpha$-plots, the black dotted line represents the average cosmic-ray background estimated in each bin by fitting a constant between the range $27^{\circ} \leq \alpha \leq 72^{\circ}$. The average background event rate per bin estimated from the procedure mentioned above is $\sim 50.1 \pm 1.0$ per hour. As TACTIC has observed Mrk 421 for 12 consecutive days during the above mentioned period (MJD 56001 - 56013) and detected the source with relatively higher statistical significance at $\mathrm{TeV}$ energies and based on the indications of comparatively higher flux state of the source from the other wavelengths during that time, we separately studied the spectral behavior of the source during this period.

The observed average excess event rate is $\sim 15.2 \pm 2.0$ per hour during this period whereas during the whole period of observation (MJD 55958 - 56044) excluding the period MJD $56001-56013$, the average rate comes out to be $\sim 9.74 \pm 2.65$ per hour. An ANN-based energy reconstruction procedure has been used to determine the differential spectrum of the source during 15 - 26 March, 2012 [46]. A powerlaw function of the form $d N / d E=N_{0} \times\left(E / 10^{3} G e V\right)^{-\Gamma}$ has been used to fit the observed spectrum which yields the values of normalization $N_{0}$ and spectral-index $\Gamma$ to be $(2.47 \pm 0.48) \times 10^{-14} \mathrm{ph} \mathrm{cm}^{-2} \mathrm{~s}^{-1} \mathrm{GeV}^{-1}$ and $2.58 \pm 0.22$ respectively having a $\chi^{2} /$ ndf $\sim 1.21 / 5$ (Figure 4 ). 

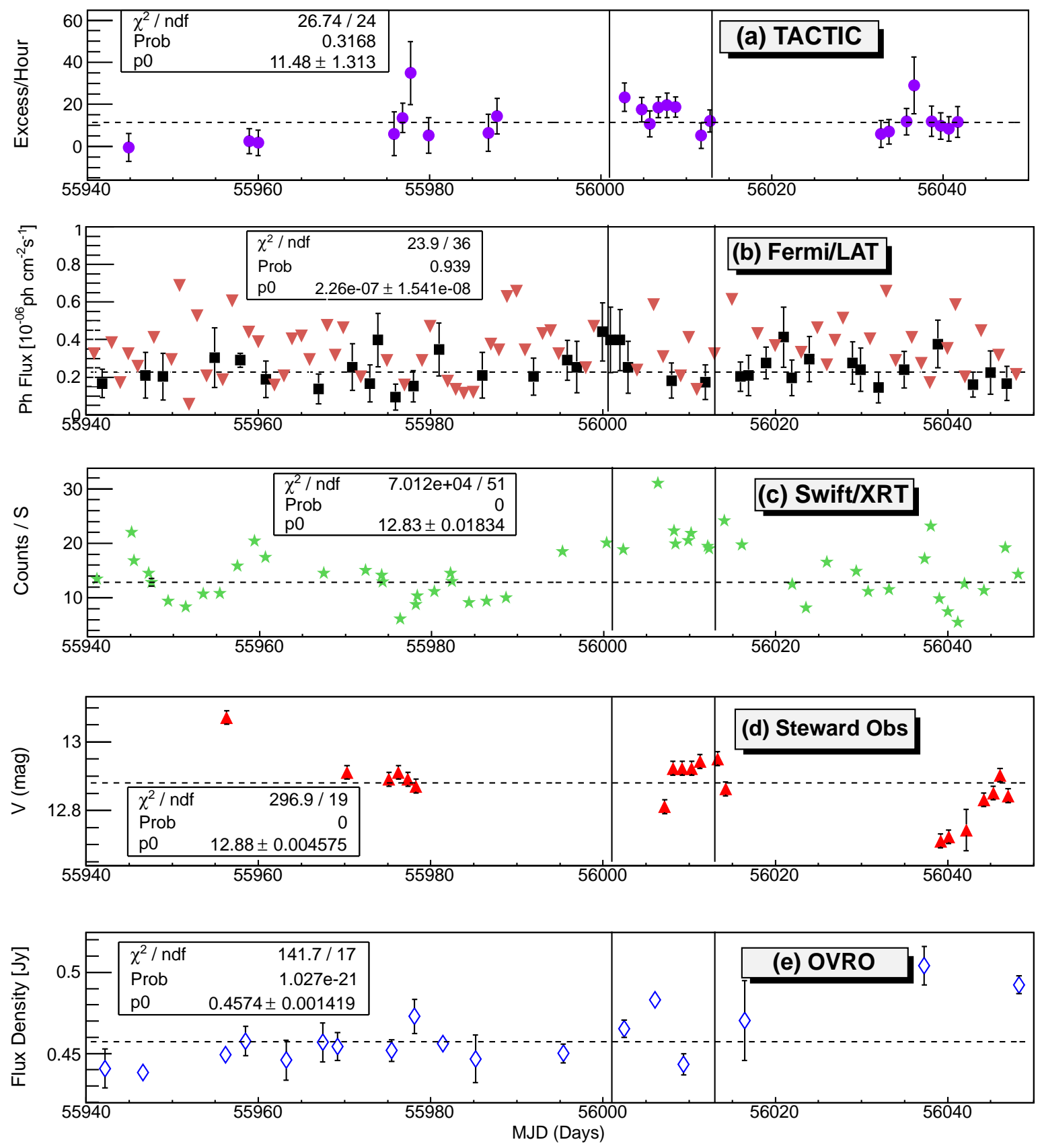

Fig. 3. Light curves of Mrk 421 from VHE to radio during 01 February - 26 April, 2012 (MJD 55958 - 56044). (a) Daily averaged VHE $\gamma$-ray rate (excess event rate) observed with TACTIC. (b) Fermi/LAT light curve with one-day bin presented with black points. The red lower triangles represent the upper-limit on the flux. (c) Swift/XRT count rates are shown with green stars. (d) and (e) represent the optical and radio light-curves respectively. The period of MJD 56001 - 56013 is shown with the vertical lines in the figure. 


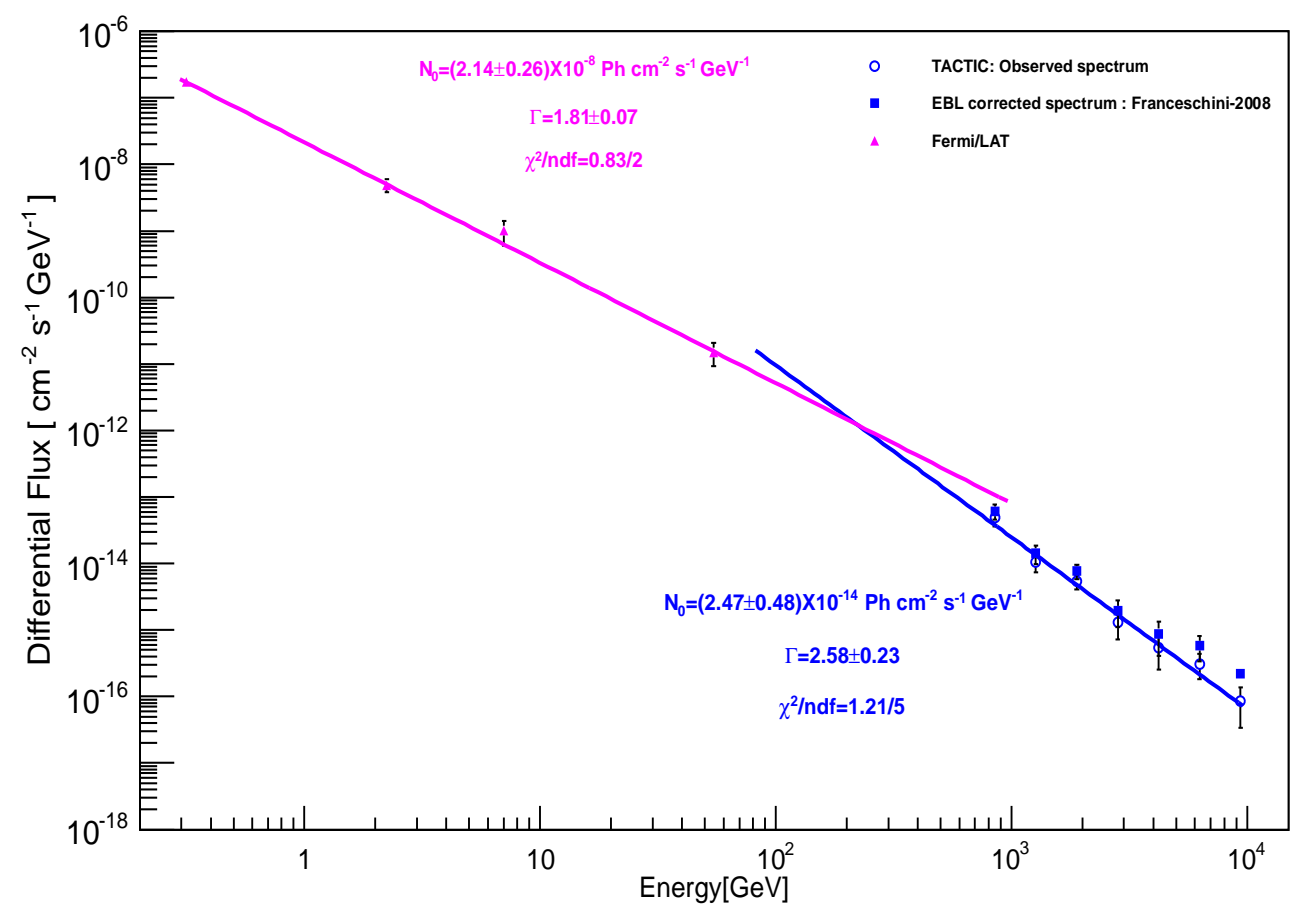

Fig. 4. TACTIC and Fermi/LAT-measured Mrk 421 differential energy spectrum averaged over the period 15 to 26 March, 2012. These two spectra are fitted separately with power-law function. The fitted values of the parameters i.e. power-law index and the normalization are given in the figure.

Table 3

Differential energy spectrum flux points of Mrk 421 observed with TACTIC during 15 - 26 March, 2012.

\begin{tabular}{ccc}
\hline $\begin{array}{c}\text { Mean Energy } \\
(\mathrm{TeV})\end{array}$ & $\begin{array}{c}\text { Flux } \\
\left(\mathrm{cm}^{-2} \mathrm{TeV}^{-1} \mathrm{~s}^{-1}\right)\end{array}$ & $\begin{array}{c}\text { Flux Error } \\
\left(\mathrm{cm}^{-2} \mathrm{TeV}^{-1} \mathrm{~s}^{-1}\right)\end{array}$ \\
\hline 0.85 & $4.88 \times 10^{-11}$ & $1.24 \times 10^{-11}$ \\
1.26 & $1.06 \times 10^{-11}$ & $3.25 \times 10^{-12}$ \\
1.89 & $5.37 \times 10^{-12}$ & $1.27 \times 10^{-12}$ \\
2.82 & $1.29 \times 10^{-12}$ & $5.72 \times 10^{-13}$ \\
4.21 & $5.42 \times 10^{-13}$ & $2.89 \times 10^{-13}$ \\
6.28 & $3.06 \times 10^{-13}$ & $1.26 \times 10^{-13}$ \\
9.36 & $8.59 \times 10^{-14}$ & $5.21 \times 10^{-14}$ \\
\hline
\end{tabular}




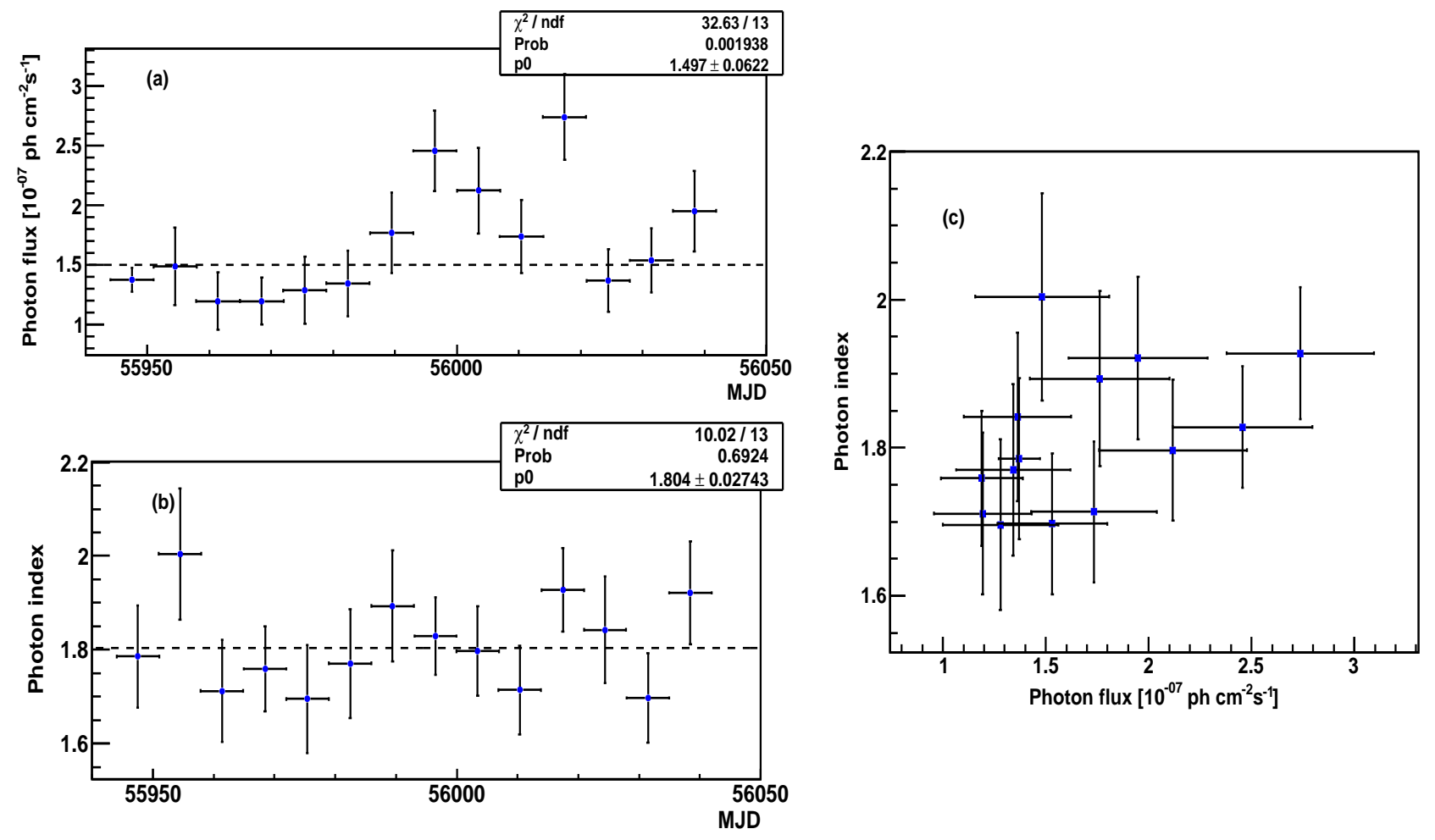

Fig. 5. (a) Fermi/LAT light curve of Mrk421 with 7 days bin for the period 19th January to 26 April, 2012 and (b) the corresponding photon index from power-law fit in the energy range $0.1-300 \mathrm{GeV}$. (c) The scatter plot of photon index with flux.

\subsection{HE results}

The daily light curve of Fermi/LAT observations of Mrk 421 in the energy band 0.1 - $300 \mathrm{GeV}$ using the archival analyzed data collected during 01 February to 26 April, 2012 (MJD 55958 - 56044) is shown in Figure 3(b). The upper limits (TS $<25)$ on the integrated fluxes are shown by downward red lower-triangles whereas the integrated fluxes $(\mathrm{TS} \geq 25)$ are shown by filled black squares. The dashed line gives the average photon flux obtained with a constant fit which is $(2.3 \pm 0.1) \times 10^{-7} \mathrm{ph} \mathrm{cm}^{-2} \mathrm{~s}^{-1}$ with a $\chi^{2} /$ ndf $\sim 24 / 36$. In order to improve the statistics, we have analyzed the data with a bin size of seven days. The results based on seven-days time bin are shown in Figure 5(a) and 5(b) which show the variation of $\gamma$-ray photon flux in the $0.1-300 \mathrm{GeV}$ energy range and the corresponding variation in the photon index from a power-law fit during this period respectively. Figure 5(c) shows the scatter plot of flux vs index. It is very clear from the Figure 5(a) that the source shows some flux enhancement between MJD 55985 to 56015 which includes the period 15 - 26 March, 2012 (MJD 56001 - 56013). The photon-index does not show significant variations during our observation period as can be seen in Figure 5(b). To study the source behavior in the shorter time scale we analyzed the data during MJD 55985 to 56015 with 2 days binning and the results are shown in Figure 6. The variability in the flux and the photon index are very much evident 


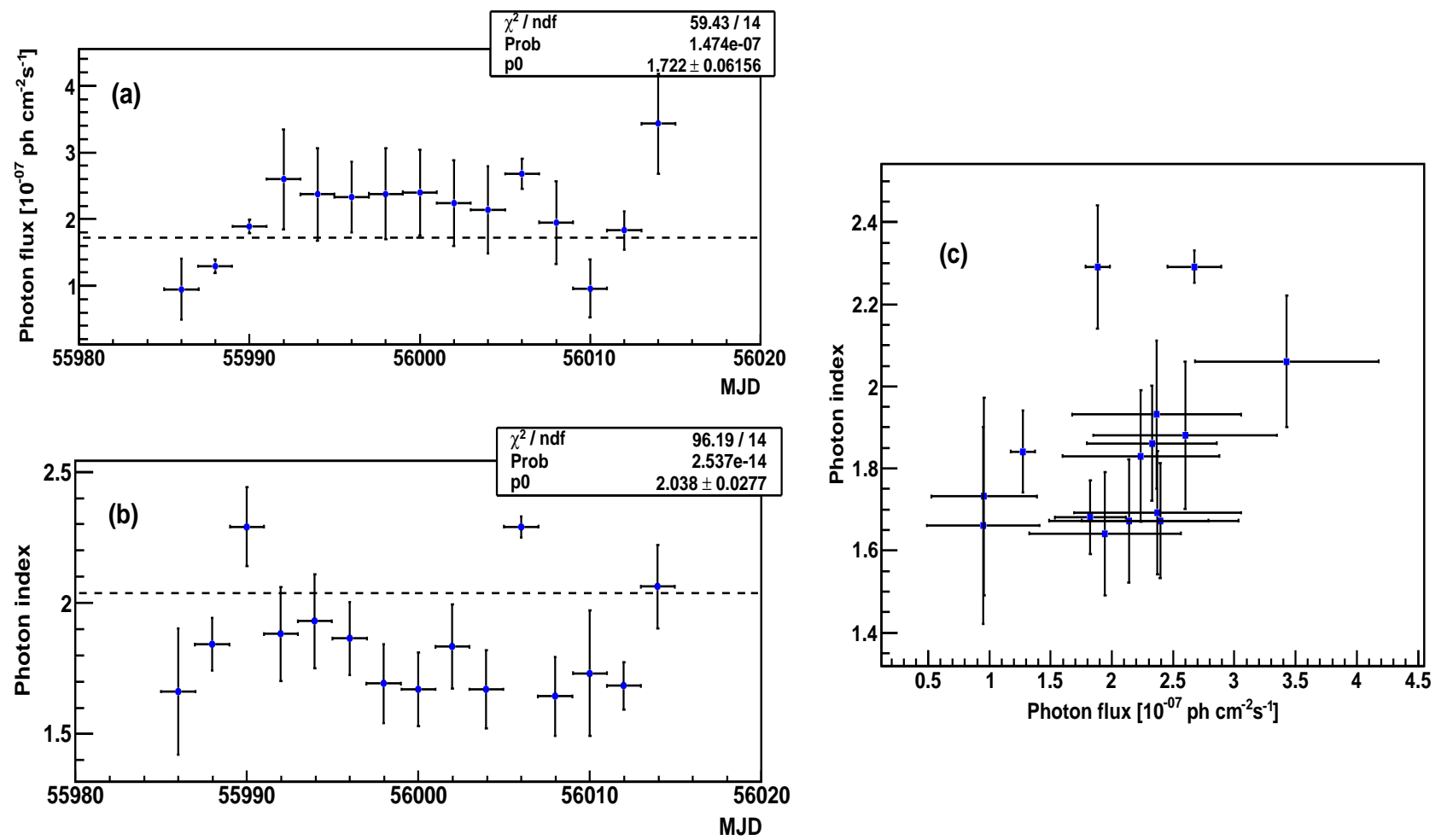

Fig. 6. (a)Fermi/LAT light curve of Mrk 421 with 2 days bin and (b) the corresponding photon index from power-law fit for the period MJD 55985 to 56015 in the energy range $0.1-300 \mathrm{GeV}$. (c) The scatter plot of photon index with flux.

from the figures 6(a) and 6(b) respectively. The statistically significant variability in the flux and photon index during this period are also supported by the $\chi^{2} / \mathrm{ndf}$ values, shown in the figures, when fitted with a constant. Figure 6(c) is a scatter plot of flux vs photon index during this short period which does not show any obvious correlation between photon flux and index. Details of the correlation studies are given in section 4.6.

The $\gamma$-ray photon fluxes were also estimated in four sub energy bands: $0.1-1.0 \mathrm{GeV}$, 1.0 - 5.0 GeV, 5.0 - 10.0 GeV, 10.0 - 300.0 GeV to obtain the spectrum. For each energy bin all the sources in the background model were fixed to the value obtained over the whole energy range (e.g. 0.1 - $300.0 \mathrm{GeV}$ ). We determine the HE differential $\gamma$-ray spectrum and fit with power-law function in the energy range 0.1-300 GeV for the period 15 - 26 March, 2012 (MJD 56001 - 56013) as shown in Figure 4(with the pink line). The fitted value of normalization $N_{0}$ and power-law index $\Gamma$ are $(2.14 \pm 0.26) \times 10^{-8} \mathrm{ph} \mathrm{cm}^{-2} \mathrm{~s}^{-1} \mathrm{GeV}^{-1}$ and $1.81 \pm 0.07$ respectively. The photon fluxes are also converted into energy flux for $\nu F_{\nu}$ representation to derive the SED for the period which is nearly simultaneous with TACTIC observation [Figure 9]. 
The count rates in the archival X-ray data obtained with Swift/XRT detector for Mrk 421 between the energy range 0.3 to $10.0 \mathrm{keV}$ are shown in Figure 3(c) for whole observation period corresponding to the TACTIC observation. The daily binned light curve is characterized by a very large value of $\chi^{2} /$ ndf $\sim 70920 / 52$ with respect to the fitted constant value of $12.40 \pm 0.02$ counts/s (represented by the dotted line in the plot). The corresponding probability that the light curves is consistent with steady X-ray emission, is approaching zero, thereby indicating the variability of the source at X-ray energies. To produce the simultaneous SED with TACTIC observation, we separately analyzed the Swift/XRT data of the duration 15 - 26 March, 2012 (MJD 56001 - 56013) following the the procedure mentioned in section 3.3. The details of flux and index derived for the total and sub-energy bands are given in the Table 4. 
Table 4

Swift analysis of Mrk 421 during 15 - 26 March, 2012

\begin{tabular}{|c|c|c|c|c|c|c|c|c|c|}
\hline Obs ID & Date & $\chi_{\nu}^{2}(\mathrm{ndf})$ & $\begin{array}{l}\text { Power-law } \\
\text { Index }\end{array}$ & $\begin{array}{c}\text { Normalization } \\
\text { ph } \mathrm{keV}^{-1} \mathrm{~cm}^{-2} \mathrm{~s}^{-1}\end{array}$ & \multicolumn{5}{|c|}{ Flux $\left(10^{-10} \mathrm{ergs} \mathrm{cm}^{-2} \mathrm{~s}^{-1}\right)$} \\
\hline 00091137078 & $16-03-12$ & $1.696(145)$ & $2.539_{-0.013}^{+0.014}$ & $0.117_{-0.001}^{+0.002}$ & $5.248_{-0.045}^{+0.047}$ & $2.265_{-0.017}^{+0.023}$ & $1.489_{-0.013}^{+0.013}$ & $0.895_{-0.007}^{+0.009}$ & $0.597_{-0.006}^{+0.004}$ \\
\hline 00091137080 & $20-03-12$ & $1.009(216)$ & $2.161_{-0.010}^{+0.009}$ & $0.204_{-0.002}^{+0.001}$ & $9.954_{-0.073}^{+0.056}$ & $2.938_{-0.017}^{+0.020}$ & $2.679_{-0.019}^{+0.015}$ & $2.244_{-0.015}^{+0.014}$ & $2.084_{-0.014}^{+0.013}$ \\
\hline 00031202109 & $23-03-12$ & $1.281(170)$ & $2.434_{-0.011}^{+0.012}$ & $0.141_{-0.001}^{+0.001}$ & $6.368_{-0.058}^{+0.044}$ & $2.506_{-0.017}^{+0.248}$ & $1.803_{-0.017}^{+0.013}$ & $1.189_{-0.009}^{+0.010}$ & $0.867_{-0.006}^{+0.008}$ \\
\hline 00091137083 & $24-03-12$ & $1.150(183)$ & $2.332_{-0.011}^{+0.011}$ & $0.142_{-0.001}^{+0.001}$ & $6.531_{-0.049}^{+0.046}$ & $2.328_{-0.018}^{+0.016}$ & $1.828_{-0.013}^{+0.014}$ & $1.318_{-0.008}^{+0.012}$ & $1.054_{-0.008}^{+0.008}$ \\
\hline 00091137085 & $26-03-12$ & $1.005(162)$ & $2.356_{-0.013}^{+0.013}$ & $0.128_{-0.002}^{+0.001}$ & $5.848_{-0.052}^{+0.045}$ & $2.133_{-0.015}^{+0.020}$ & $1.641_{-0.013}^{+0.015}$ & $1.161_{-0.010}^{+0.009}$ & $0.906_{-0.007}^{+0.008}$ \\
\hline
\end{tabular}



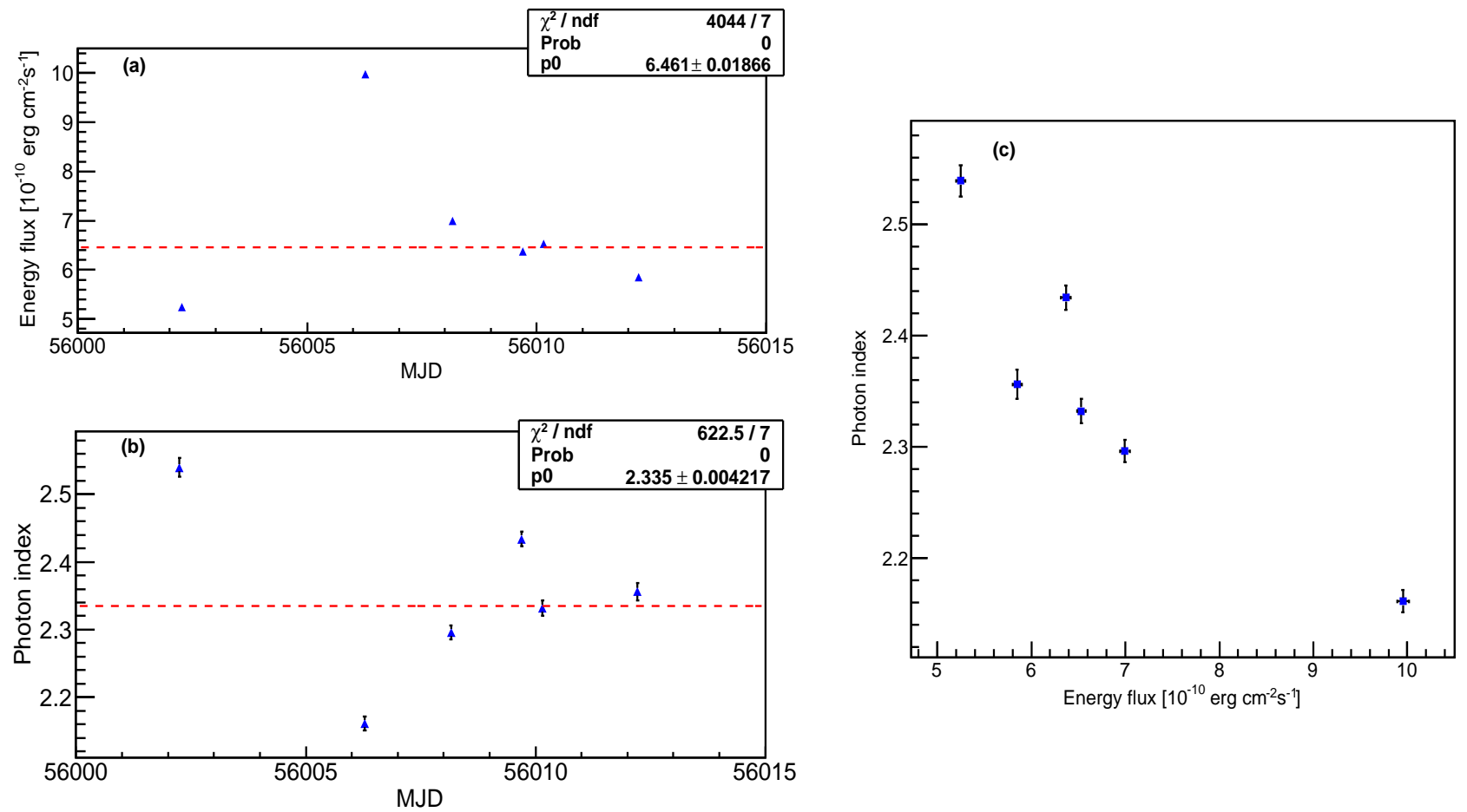

Fig. 7. (a) Swift/XRT daily light curve of Mrk421, (b) the corresponding photon index from power-law fit for the period MJD $56001-56013$ in the energy range $0.3-10.0 \mathrm{keV}$ and (c) the scatter plot of photon index with flux.

The flux and photon-index variations during this period are shown in Figure 7. Since there are two observations on the 22 and 26 March, 2012, we used the points with higher exposure time in the light curve. Details of the analysis are given in the Table 4. We notice that the flux level on 20 March, 2012 (MJD 56006) was considerably higher than the average flux level during this period. This sudden enhancement of X-ray flux is observed in all the four sub-energy bands mentioned in Table 4 . The scatter plot of X-ray flux vs photon index obtained with power-law fit as shown in Figure 7(c), also indicates correlation between these two quantities. Detailed discussions are given in section 4.6.

\subsection{Optical and Radio results}

The archival data set of optical fluxes of the source in V-band obtained from Steward Observatory are shown in the Figure 3(d). The light curve is characterized by $\chi^{2}$ /ndf value $\sim 296 / 19$ with respect to a constant fit of $12.880 \pm 0.005$ magnitude (V band) for the whole observation period 01 February to 26 April, 2012 (MJD 55958 - 56044). The probability that the optical fluxes are consistent with a steady emission is $<0.0001 \%$, indicating the flux variability in the optical waveband of the source as well. 
The archival radio data of OVRO are also shown in the Figure 3(e). The average radio flux obtained with a constant fit during the period 01 February to 26 April, 2012 (MJD $55958-56044$ ) is $0.457 \pm 0.001$ Jy with a $\chi^{2} /$ ndf $\sim 141 / 17$. This indicates that the probability of variability in the radio flux of the source during our observation is $>99.999 \%$.

\subsection{Flux variability}

In order to characterize and quantify the flux variability in different energy bands, we followed the approach given in [55] to calculate the fractional variability $\left(\mathrm{F}_{\mathrm{var}}\right)$ defined as

$$
F_{v a r}=\frac{\sqrt{S^{2}-\left\langle\sigma_{e r r}^{2}\right\rangle}}{\left\langle F_{\nu}\right\rangle}
$$

where $\left\langle F_{\nu}\right\rangle$ is mean photon flux, $S$ is standard deviation and $\sigma_{e r r}^{2}$ is mean squared error of $\mathrm{N}$-flux points in the light curve. We estimate the uncertainty in $\mathrm{F}_{\text {var }}$ as

$$
\Delta F_{v a r}=\frac{1}{F_{v a r}} \sqrt{\frac{1}{2 N}} \frac{S^{2}}{\left\langle F_{\nu}\right\rangle^{2}}
$$

As the daily averaged light curves (Figure 3) of Mrk 421 have already given indication of variability in flux in most of the energy bands, we calculate the values of $F_{\text {var }}$ and errors for each energy band for the whole observation period (MJD 55958 - 56044) which are shown in Figure 8. The $\mathrm{F}_{\text {var }}$ in the HE domain is calculated using seven days binned Fermi/LAT light curve [Figure 5(a)] as most of the daily averaged flux points in this energy band are not significant $(\mathrm{TS}<25)$. We obtain the maximum fractional variability in the X-ray band is $0.36 \pm 0.04$, which is a characteristic signature of the source consistent with recent results given in[61].

\subsection{Spectral evolution and correlation}

Typically blazars show systematic variation in the spectral shape during flares [62]. Sometimes photon index vs flux diagram shows loops during the course of flare, which might be related to the dynamics of the system [63]. During 15 - 26 March, 2012 (MJD 56001 - 56013), we observe this kind of systematic variation between photon index and X-ray flux detected by Swift/XRT in the energy band 0.3 to 10.0 $\mathrm{keV}$. In Figure 7(c) one can clearly see that the X-ray spectra harden when Xray flux increases. So we can confirm the typical harder-when-brighter behavior in the X-ray band is visible even when the source is in comparatively high state. We characterize the correlation with Pearson correlation coefficient which turns out to be $-0.87 \pm 0.20$. It shows significant anti-correlation between X-ray flux and index. 


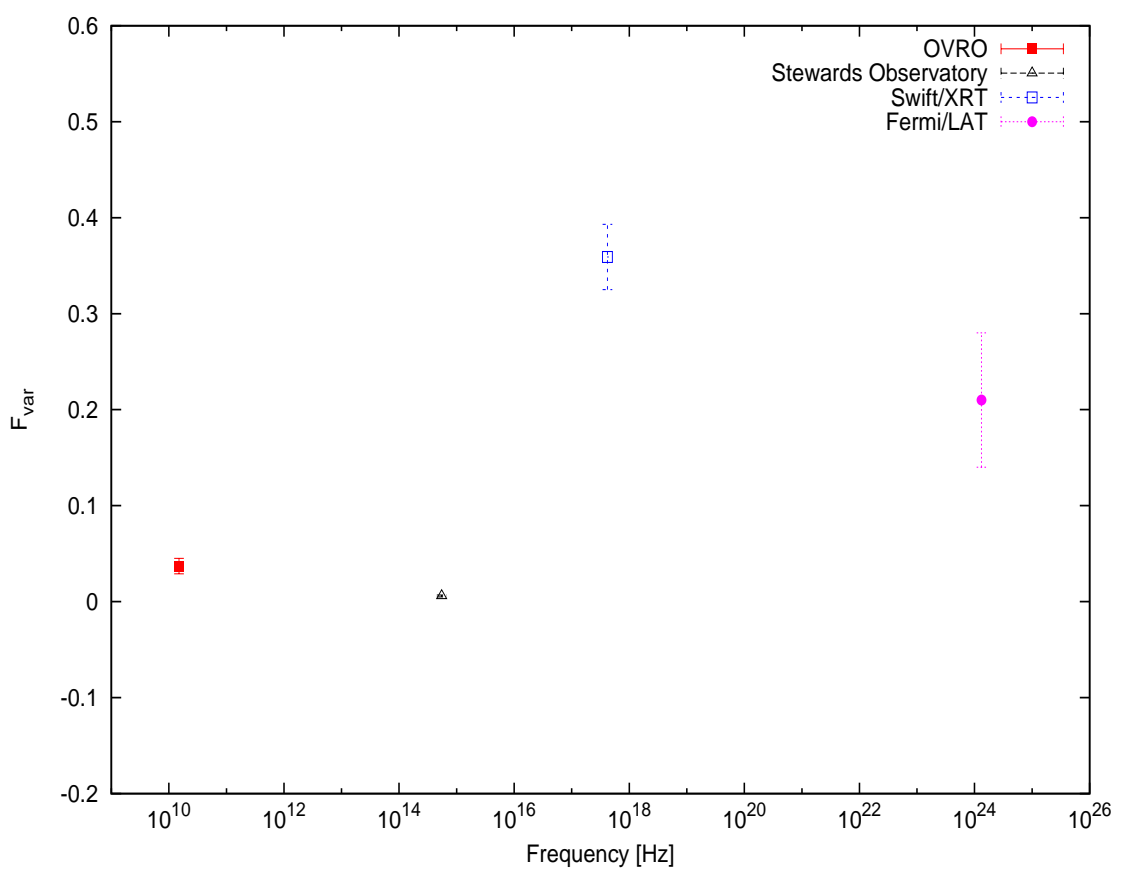

Fig. 8. Fractional variability $F_{v a r}$ as a function of frequency measured by different instruments.

We also studied the temporal evolution of the spectral points shown in Figure 7(c) in search of clockwise or anti-clockwise loop pattern, but did not find any prominent pattern during this period.

We study the correlation between flux and index in the energy range $0.1-300.0$ $\mathrm{GeV}$ also with weekly binned as well as 2 days binned light curves of Fermi/LAT data. In both the cases, the scatter plots of flux and photon index shown in Figure 5(c) and Figure 6(c), do not show any correlated variations of these two quantities. The Pearson correlation coefficients for the 7 days and 2 days binned Fermi/LAT light curves are $0.45 \pm 0.26$ and $0.40 \pm 0.25$ respectively, which also imply no significant correlations between HE flux and index in longer or shorter time duration.

\section{Spectral modeling during 15 - 26 March, 2012}

The broadband SED of Mrk 421 during 15 - 26 March, 2012 is shown in Figure 9. The SED is modeled using synchrotron and SSC process[65]. This model assumes a spherical emission region of radius $R$ filled with relativistic electrons with uniform density distribution. The region moves down the jet with a bulk Lorentz factor $\Gamma$ at an angle $\theta$ with the line of sight. Special relativistic effects are described by the Doppler factor, $\delta=[\Gamma(1-\beta \cos \theta)]^{-1}$. The energy distribution of the emitting electrons is described by a broken power-law between minimum and maximum energy $\left[E_{\min }, E_{\max }\right]$, with the slopes $n_{1}$ and $n_{2}$ below and above the break energy $E_{b}$ respectively. The electrons lose their energy by synchrotron process in a 


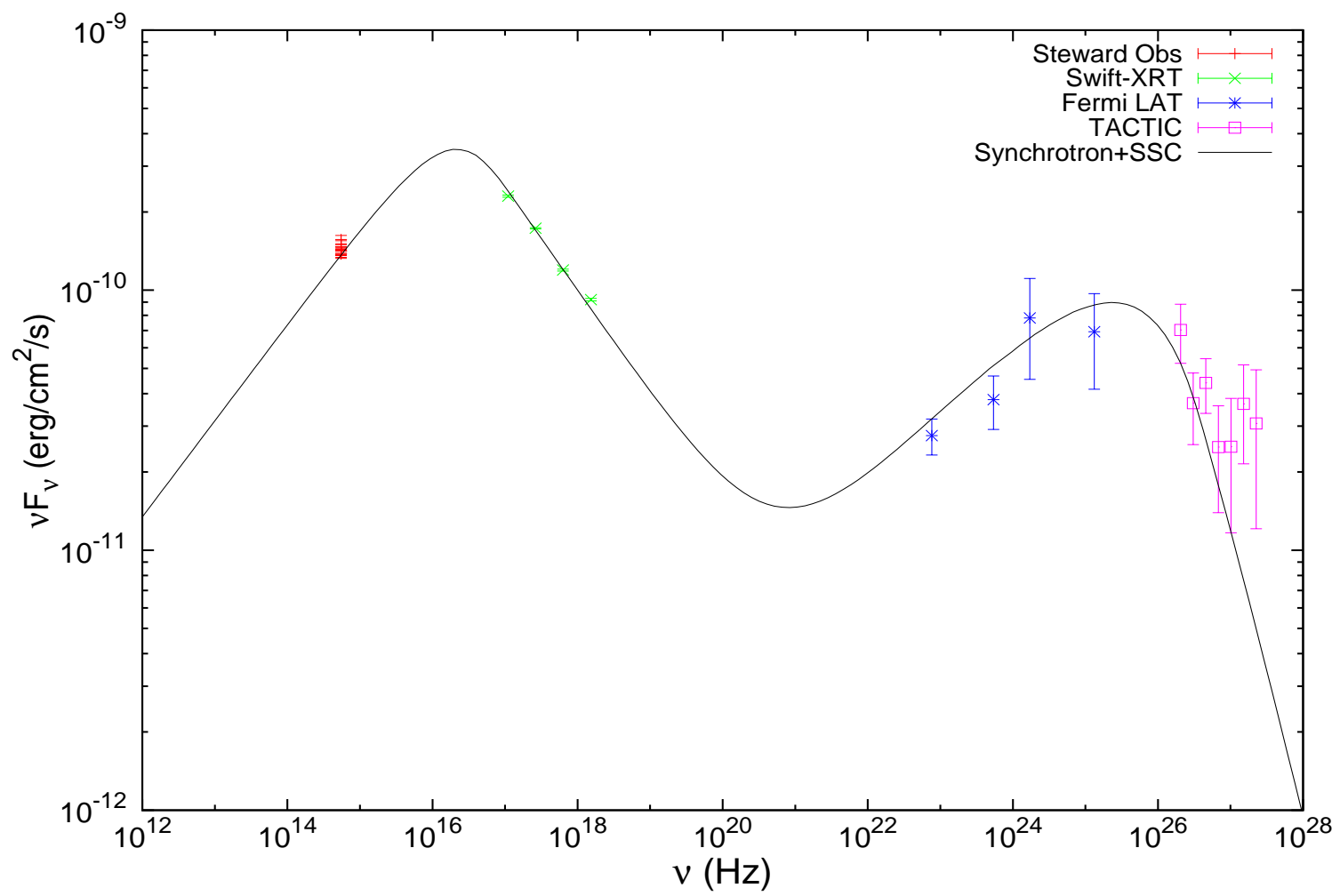

Fig. 9. Multiwavelength SED of Mrk421 for the period 15 - 26 March, 2012.

tangled magnetic field $B$ and through IC scattering of synchrotron photons itself. The total power emitted by these mechanisms in the frame of the emission regions is then transformed to the observer's frame by considering the cosmological and relativistic effects[64].

The observed flux on the Earth depends on mainly seven parameters namely, the particle energy density $u_{e}$ in the jet frame, power-law indices $n_{1}$ and $n_{2}$ of the particle distribution, the break energy $E_{b}$, the magnetic field $B$, the emission region size $R$ and the Doppler factor $\delta$. The minimum and maximum energy of the particle distribution $E_{\min }$ and $E_{\max }$ are weak parameters and only decides the minimum and maximum frequency of the emitted photons. We found that the broadband SED of Mrk 421 can be well reproduced by this simple model and in Figure 9 we show the observed fluxes along with the model spectrum. The observed VHE spectrum at the source is corrected for EBL (Extragalactic background light) absorption using the model described by [66]. In Table 5 we present the physical parameters corresponding to the model spectrum.

\section{Discussion and Conclusion}

In this paper, apart from the study of a high flux state of blazar Mrk 421 observed at $\mathrm{TeV}$ energies using TACTIC $\gamma$-ray telescope, we also present nearly simultaneous 
Table 5

SSC model parameters used to model SED.

\begin{tabular}{ll}
\hline Parameter & Value \\
\hline$\delta$ & 32.1 \\
$B$ & $0.015 \mathrm{G}$ \\
$R$ & $8.0 \times 10^{16} \mathrm{~cm}$ \\
\hline$E_{\min }$ & $2.0 \times 10^{7} \mathrm{eV}$ \\
$E_{\max }$ & $5.0 \times 10^{14} \mathrm{eV}$ \\
$E_{b}$ & $6.2 \times 10^{10} \mathrm{eV}$ \\
$n_{1}$ & 2.3 \\
$n_{2}$ & 3.8 \\
$u_{e}$ & $0.77 \times 10^{-3} \mathrm{erg} / \mathrm{cm}^{3}$ \\
\hline
\end{tabular}

observation of the source in HE with Fermi/LAT, X-ray with Swift/XRT, optical with Steward Observatory and radio with OVRO.

Detailed analysis of the TACTIC data recorded during 15 - 26 March, 2012 the source had exhibited a possible high emission state which is evident from the detection of $529 \pm 76 \gamma$-like events at $6.9 \sigma$ significance above $850 \mathrm{GeV}$ energy threshold during this period. Using these events, the source's differential energy spectrum has been estimated which fits well with a power-law function of the form $d N / d E=N_{0} \times\left(E / 10^{3} \mathrm{GeV}\right)^{-\Gamma}$ yielding the values of normalization constant $\left(N_{0}\right)$ and spectral-index $(\Gamma)$ to be $(2.47 \pm 0.48) \times 10^{-14} \mathrm{ph} \mathrm{cm}^{-2} \mathrm{~s}^{-1} \mathrm{GeV}^{-1}$ and $2.58 \pm 0.22$ respectively with a $\chi^{2} /$ ndf value of $\sim 1.21 / 5$. The comparison of $\mathrm{HE}$ and VHE spectra is depicted in Figure 4 which shows a prominent change of slope in the differential energy spectrum of Mrk 421 from $\mathrm{GeV}$ to $\mathrm{TeV}$ energies. The observed difference in the slope might be attributed to Klein-Nishina effect on IC scattering of photons in the $\mathrm{TeV}$ energy range.

In order to study the variable nature of the source in different electromagnetic bands, we have estimated the fractional variability $F_{v a r}$ in the corresponding bands using daily light curves shown in Figure 3. It may be noted that in HE (Fermi/LAT) band 7 days bin has been used as in this band most of the daily averaged flux points are with TS $<25$, whereas in the VHE range the TACTIC data did not allow us to estimate $F_{v a r}$ due to lac of significance of detection of $\gamma$-like events on daily basis due to limited sensitivity of the telescope. We find that the value of $F_{v a r}$ is highest for $\mathrm{HE} \gamma$-ray data which is depicted in Figure 8. Further it is also clear from this figure that for the X-ray band $F_{\text {var }}$ turns out to be $0.359 \pm 0.034$ which is the maximum variability. In $\mathrm{HE}$ band, the value of $F_{\text {var }}$ is $0.21 \pm 0.07$ which shows less variability than X-ray band. In contrast, we do not find any significant values 
of $F_{v a r}$ for optical ( $\mathrm{V}$ band) and radio $(15 \mathrm{GHz})$ bands as is also clear from the same figure. From this study we conclude that the source exhibited a larger variability at the X-ray and $\gamma$-ray energies when compared to that at lower energies viz the optical and radio bands. Therefore these results lend support to the SSC model wherein the synchrotron emission from the high energy electrons dominate X-ray emission whereas the HE emission is produced by the low-energy electrons that inverse-Compton scatter the synchrotron photons. Further, a comparable variability in X-ray and HE indicates the intrinsic variability in the whole electron spectrum suggesting that both these emissions are coming possibly from the same zone of the source.

The recent multiwavelength study of Mrk 421 during 2009 shown in [61] reveals that the source showed little variability in radio band, and maximum variability in X-ray band in its non-flaring state which is similar with our results of having maximumum variability in X-ray. This X-ray variability is significantly larger than HE variability. Authors also claimed that these results are independent of temporal binning or uneven sampling of light curves, so this result can be considered as the characteristics of Mrk 421 low state. In the past, multiwavelength campaign of Mrk 421 reported in $[69,70]$ showed that a general trend of increasing $F_{v a r}$ with higher frequency (not in a predefined high or low state). Radio and optical bands showed little variability whereas maximum variability was observed in VHE band. Comparable variability in X-ray and VHE has been shown in [69]. We have also studied the spectral evolution of the source in X-ray and HE band. In X-ray band, we have obtained Pearson correlation coefficient to be $-0.87 \pm 0.20$ which essentially means a significant anti-correlation between X-ray flux and power-law index during MJD 56001 - 56013 [Figure 7(c)]. The spectrum becomes harder when flux increases. This 'harder-when-brighter' behavior in X-ray is also typical for Mrk 421 as described in [61]. As we do not find any prominent clockwise or anti-clockwise loop pattern in the X-ray flux and photon index diagram [Figure 7(c)], it is not possible to probe obvious particle acceleration mechanism in the source [63]. The Pearson correlation coefficients obtained in the HE band are $0.45 \pm 0.26$ and $0.40 \pm 0.25$ for the 7 days and 2 days binned Fermi/LAT light curves respectively. These values indicate the absence of any significant correlation between HE flux and index during our observations which is consistent with a previous study done by [17].

Finally, we have also studied the broadband SED during 15 - 26 March, 2012 using optical, X-ray, HE and VHE observations. We model the broadband SED of the source during this period using simple synchrotron and SSC emission processes. We find that the observed fluxes can be well reproduced by this model and the parameters obtained (given in the Table 5) are consistent with generally expected parameter for the BL Lacs objects. The prominence of the high flux state of the source is evident from the X-ray light curve on MJD 56006, whereas, the poor statistics in the VHE $\gamma$-ray prevents us from obtaining the VHE spectrum particularly on that day. With the upcoming high sensitivity $\gamma$-ray telescope Major Atmospheric Cherenkov Experiment (MACE), a detailed information about the VHE spectral 
evolution of the source would be obtained, which along with the information from other satellites and ground based telescopes, will help us to understand the physics behind the high states of the source.

\section{Acknowledgements}

The authors would like to express their gratitude to all concerned colleagues of the Astrophysical Sciences Division for their contribution towards the instrumentation and observation aspects of the TACTIC telescope. The authors would also like to thank the anonymous reviewers for their very useful suggestions. This publication makes use of the data from the Fermi/LAT detector through Fermi Science Support Center (FSSC) provided by NASA, X-ray data supplied by the UK Swift Science Data Centre at the University of Leicester, optical data from the Steward Observatory and radio data from Owens Valley Radio Observatory (OVRO). This program is supported by Fermi Guest Investigator grants NNX08AW56G, NNX09AU10G and NNX12AO93G. We acknowledge Fermi blazar monitoring program which is supported by NASA under award NNX08AW31G, and by the NSF under 0808050. 


\section{References}

[1] C.M. Urry and P. Padovani, PASP, 107 (1995) 803.

[2] R.M. Sambruna, L. Maraschi and C.M. Urry, ApJ, 463 (1996) 444.

[3] G. Fossati et al., MNRAS, 299 (1998) 433.

[4] G. Ghisellini, F. Haardt and R. Svensson, MNRAS, 297 (1998) 348.

[5] C.D. Dermer, R. Schlickeiser and A. Mastichiadis, A\&A, 256 (1992) L27.

[6] M. Sikora, M.C. Begelman and M.J. Rees, ApJ, 421 (1994) 153.

[7] K. Mannheim, Phys. Rev. D, 48 (1993) 2408.

[8] F.A. Aharonian, New A, 5 (2000) 377.

[9] M. Pohl and R. Schlickeiser, A\&A, 354 (2000) 395.

[10] A. Mücke and R.J. Protheroe, 27th International Cosmic Ray Conference, 3 (2001) 1153.

[11] A. Mücke et al., Astroparticle Physics, 18 (2003) 593.

[12] M. Punch et al., Nature, 358 (1992) 477.

[13] D.J. Macomb et al., ApJ, 449 (1995) L99.

[14] J.H. Buckley et al., ApJ, 472 (1996) L9.

[15] N. Galante, 32nd International Cosmic Ray Conference, 8 (2011) 63.

[16] V.A. Acciari et al., ApJ, 738 (2011) 25.

[17] A.A. Abdo et al., ApJ, 736 (2011) 131.

[18] J. D. Hartman et al., ApJ, 553 (2001) 683.

[19] J. Kataoka et al., ApJ, 560 (2001) 659.

[20] J.A. Zweerink et al., ApJ, 490 (1997) L141.

[21] M. Tluczykont, Proceedings of Texas symposium (2010), 197.

[22] F. Aharonian et al., A\&A, 410 (2003) 813.

[23] F. Aharonian et al., A\&A, 437 (2005) 95.

[24] J. Albert et al., ApJ, 663 (2007) 125.

[25] F. Piron et al., A\&A, 374 (2001) 895.

[26] R.C. Rannot et al., 29th International Cosmic Ray Conference, 4 (2005) 355.

[27] K.K. Yadav et al., Astroparticle Physics, 27 (2007) 447. 
[28] P. Chandra et al., Journal of Physics G Nuclear Physics, 37 (2010) 125201.

[29] G. Aielli et al., ApJ, 714 (2010) L208.

[30] B. Bartoli et al., ApJ, 734 (2011) 110.

[31] J. Aleksić et al., A\&A, 542 (2012) A100.

[32] P. Chandra et al., Journal of Physics G Nuclear Physics, 39 (2012) 045201.

[33] N. Isobe et al., PASJ, 62 (2010) L55.

[34] H. Gaur, A.C. Gupta and P.J. Wiita, AJ, 143 (2012) 23.

[35] A. Shukla et al., A\&A, 541 (2012) A140.

[36] R.A. Ong, The Astronomers Telegram, 2443 (2010) 1.

[37] K.K. Singh et al., Astroparticle Physics, 61 (2015) 32.

[38] N. Fraija et al., Proceedings of Science, (2014) 152.

[39] A. Sinha et al., A\&A, 580, (2015), A100.

[40] V. S. Paliya et al., ApJ, 811 (2015) 143.

[41] T. Hovatta et al., MNRAS, 448 (2015) 3121.

[42] K.K. Yadav et al., Nuclear Instruments and Methods in Physics Research A, 527 (2004) 411.

[43] S.R. Kaul et al., Nuclear Instruments and Methods in Physics Research A, 496 (2003) 400.

[44] R. Koul et al., Nuclear Instruments and Methods in Physics Research A, 578 (2007) 548.

[45] A. K. Tickoo et al., Pramana-J. Phys., 82 (3) (2014) 585.

[46] V. K. Dhar et al., Nuclear Instruments and Methods in Physics Research A, 606 (2009) 795.

[47] A.M. Hillas, 19th International Cosmic Ray Conference, 3 (1985) 445.

[48] A.M. Hillas et al., ApJ, 503 (1998) 744.

[49] G. Mohanty et al., Astroparticle Physics, 9 (1998) 15.

[50] M.K. Koul and M.L. Sapru, Bulletin of the Astronomical Society of India, 31 (2003) 497.

[51] T.P. Li and Y.Q. Ma, ApJ,272 (1983) 317.

[52] W.B. Atwood et al., ApJ, 697 (2009) 1071.

[53] J.R. Mattox et al., ApJ, 461 (1996) 396. 
[54] T. Alexander, Astronomical Time Series,Astrophysics and Space Science Library, 218 (1997) 163.

[55] R. Edelson et al., ApJ, 568 (2002) 610.

[56] P.S. Smith et al., Fermi Symposium, Conference Proceedings, C091122 (2009).

[57] M. S. Bessel, PASP, 91 (1979) 589.

[58] N. Gehrels et al., ApJ, 611 (2004) 1005.

[59] P. Nolan et al., ApJS, 199 (2012) 31.

[60] J. L. Richards et al., ApJS, 194 (2011) 29.

[61] J. Aleksić et al., A\&A, 576 (2015) A126.

[62] G. Fossati et al., ApJ, 541 (2000) 166.

[63] J. Kirk et al., Astroparticle Physics, 11 (1999) 45.

[64] M. C. Begelman et al., Nature, 287 (1980) 307.

[65] S. Sahayanathan et al., MNRAS, 419 (2012) 1660.

[66] A. Franceschini et al., A\&A, 487 (2008) 837.

[67] P. M. Kalberla et al., A\&A, 440 (2005) 775.

[68] A. Burrows et al., Space Sci. Rev., 120 (2005) 165.

[69] D. Horan et al., ApJ, 695 (2009) 596.

[70] M. Błażejowski et al., ApJ, 630 (2005) 130.

[71] A. Sinha et al., A\&A, 591 (2016) 83.

[72] B. Bartoli et al., ApJS, 222 (2016) 6. 